\title{
Téoros
}

Revue de recherche en tourisme

\section{État des lieux des festivals en France}

\section{Luc Benito}

Volume 21, numéro 1, printemps 2002

URI : https://id.erudit.org/iderudit/1071539ar

DOI : https://doi.org/10.7202/1071539ar

Aller au sommaire du numéro

Éditeur(s)

Université du Québec à Montréal

ISSN

0712-8657 (imprimé)

1923-2705 (numérique)

Découvrir la revue

Citer cet article

Benito, L. (2002). État des lieux des festivals en France. Téoros, 21(1), 48-56. https://doi.org/10.7202/1071539ar d'utilisation que vous pouvez consulter en ligne.

https://apropos.erudit.org/fr/usagers/politique-dutilisation/ 


\section{État des lieux des festivals en France}

\section{Luc Benito}

This article presents the status of the festivals in France. It first gives details on the notion of festival, as it is presently too vague due to the range of events of that kind. We will see that the concept of festival in France really appeared in the beginning of the wentieth century as a particularly relevant caltural medium for the artistic circles. It is however in other sectors, such as tourism, communication, or local animation, that its efficiency led, a linle later, the authorities to emphasise its development. We will then see that this activity, albeit its very strong growth in the $80^{\prime}$ 's, paradoxically undergoes a certain number of constraints, especially relative to financing. The conjunction of the events and arts domains actually leads to uncertainty. Finally, we will make certain recommendations, with regard to that uncertainty. a France a connu une explosion du nombre des festivals dans les années 1980, au point de parler de véritable phénomène. Comme la plupart des activités culturelles en France, les festivals sont dépendants de l'intervention financière des pouvoirs publics. Ces politiques culturelles, pourtant enviees dans le monde entier, suscitent beaucoup de réserves car elles sont parfois à l'origine d'effets pervers qui nuisent au fonctionnement des entreprises du secteur.

Loin de remettre en question un systeme dont les vertus sont innombrables, notamment du point de vue de l'art stricto sensu, force est de constater qu'il s'agit d'un système encore largement auto-centré. La question du et des publics n'apparaît parfois pas comme une priorité aux yeux des directeurs des structures de diffusion culturelle (musées, thétres, salles de concerts...). Les notions de marketing, de management, d'efficacité ou de rentabilité sont bannies au nom d'une indépendance par rapport aux lois du marché et aux contingences financières.

Il n'est par conséquent pas étonnant de voir l'antinomie entre le secteur du tourisme et de la culture où les premiers sont qualifiés de marchands du temple par les seconds assimilés, eux, à de vulgaires saltimbanques. Bien que quelque peu caricatural, le clivage est bien réel et se remarque même au niveau des pouvoirs publics, comme le prouve l'échec des tentatives de rapprochement entre les ministères concernés depuis une vingtaine d'années.

Bien évidemment, seules les activités culturelles à vocation touristique peuvent souffrir de cette situation. En effet, en France, le principe de « mise en tourisme $p$ des festivals, des musées et de toutes les activités de valorisation du patrimoine reste encore souvent ignoré des décideurs et des responsables de structures. Sur ce plan, la France a un retard considerable par rapport au continent nord-américain.

Forte de tels constats, la description du champ des festivals en France proposée ici se fait du point de vue de l'économiste passionné par le domaine des arts et de la culture, qui tente de savoir comment une activité comme celle des festivals - non rentable et cumulant les difficultés de fonctionnement - a pu tant se développer. Est-ce que les festivals satisfont des missions uniquement dans le domaine culturel ou sont-ils devenus aussi des instruments de développement particulièrement efficaces dans d'autres domaines (tourisme, communication...) ?

Sous la forme d'un état des lieux, la première partie du présent article donne un aperçu du phénomène avec sa définition, son histoire, sa mesure et l'Évolution de la notion, alors que la seconde s'intéresse plus spécifiquement au « système festival $b$ où l'on trouve un diagnostic de l'activité ainsi que des orientations et des perspectives de développement possibles.

\section{Le phènomène festival}

\section{La définition : une acception floue}

Tout d'abord en ce qui concerne l'utilisation du terme, la France et la plupart des pays européens se distinguent des Anglo- 
saxons (Amérique du Nord, Royaume-Uni et pays du Commonwealth) qui désignent par « festivals * toutes les fêtes à caractère folklorique, tandis que ce qu'ils appellent $\propto$ art festivals $*$ correspond davantage à ce que nous entendons par festivals.

La notion de festival pose problème en France car, à la suite de l'engouement des pouvoirs publics locaux pour l'événementiel, l'utilisation abusive du terme $\alpha$ festival s pour désigner notamment de simples événements culturels rend désormais difficile toute définition. Cette difficulté découle du fait que la notion de festival intègre deux types de critères.

Des critères objectifs facilement identifiables et qui peuvent se résumer en général par la règle des trois unités du théattre classique :

- l'unité de temps sur une courte durée et à périodicité renouvelée,

- l'unité de lieu limitée à un ou à plusieurs sites, souvent prestigieux, d'une ville,

- I'unité d'action ou de thème représentée par une discipline artistique.

Des critères subjectifs plus difficiles à appréhender d'un point de vue statistique car ils font référence à un état d'esprit :

- la célébration publique qui présente un festival comme un quasi-pèlerinage réunissant tous les passionnés d'une discipline artistique, amateurs ou professionnels, dans une communion d'esprits,

- la célébration d'un art qui induit une programmation de choix pour le directeur artistique et une prestation de qualité pour des artistes très souvent « sublimés » par l'événement.

Une telle définition des festivals intègre donc à la fois une dimension technique qui fait du festival un moment unique, mais aussi une dimension davantage qualitative qui lui confère son caractère exceptionnel et qui permet véritablement de le distinguer d'une simple série de manifestations programmées sur quelques soirs.

Un festival pourrait par conséquent se définir comme une forme de fête unique, célébration publique d'un genre artistique dans un espace-temps réduit. Suivant la rigidité de la définition, on peut grossièrement situer le nombre de festivals en France de 600 aे 2000 manifestations chaque année.

\section{L'histoire : quatre générations}

Une mise en perspective historique met en évidence quatre périodes dans lesquelles des types de festivals bien spécifiques ont fait leur apparition.

\section{Les festivals des \& Beaux-Arts *}

On trouve la première trace d'un festival en 1724 dans la manifestation d'un genre nouveau lancé en Angleterre par le Chan- celier Thomas Bisse, sorte de tournoi de musique de chambre appelé « The Three Choirs Festival w. C'est pourtant en Allemagne, à la fin du XVIII" siècle, qu'il faut situer la véritable origine des festivals. A l'époque, les plus grandes sociétés chorales d'Allemagne se rassemblaient périodiquement dans certaines grandes villes, pendant deux ou plusieurs jours, pour célébrer de grands musiciens (par exemple: le Festival de Vienne en 1811 donné en l'honneur de Haydn).

Depuis leur origine jusqu'au début du $X^{\mathrm{c}}$ siècle, les festivals étaient généralement créés sur l'initiative des grandes cours européennes, passant progressivement des concours de chant aux concerts classiques,

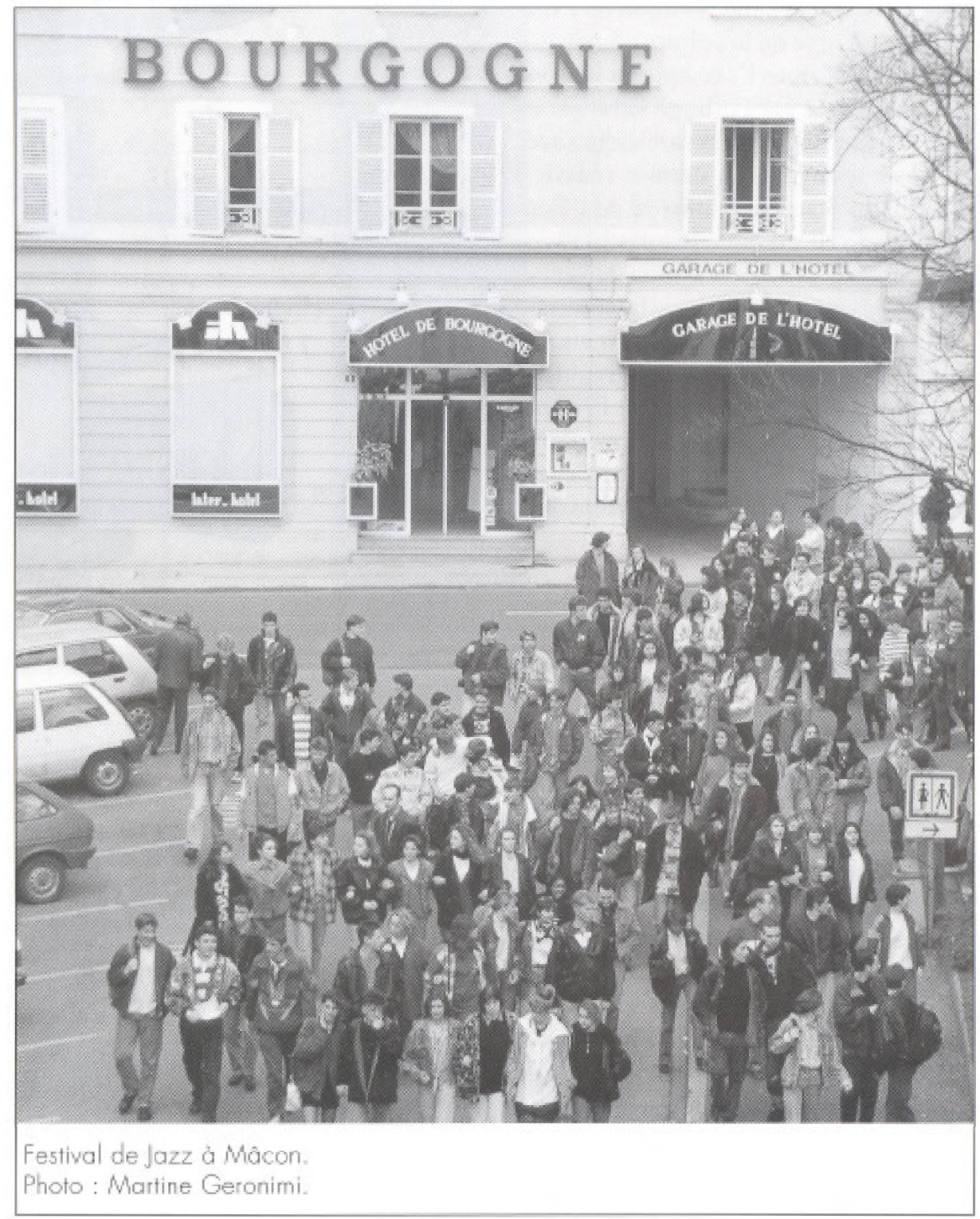


à l'image de Bayreuth crée en 1876 par Louis II de Bavière, dont beaucoup s'accordent à dire qu'il inaugure véritablement l'ère des festivals. La création de ces fêtes à caractère aristocratique, généralement dans des lieux de villégiature, se faisait dans la pure tradition du mécénat du * prince $\%$.

\section{Les festivals de promotion artistique}

Au début du $X X^{e}$ siècle apparaissent des festivals initiés généralement par des artistes, moyens pour eux de promouvoir leur discipline artistique, souvent en quête de reconnaissance publique. L'État va jouer un rôle déterminant, par sa contribution financière, cependant moins désintéressée que celle du \& prince $»$. En effet, e'est à cette époque que l'intervention de l'État dans le domaine de la culture commence à s'affirmer. Citons l'exemple du Festival de Cannes créé par Philippe Erlanger en 1946. Chargé des échanges artistiques avec les pays étrangers, ce dernier réussit à convaincre Jean Zay, ministre de l'Éducation Nationale, d'organiser dès 1939 une contre-manifestation à la Mostra de Venise, imprégnée alors de l'idéologie fasciste, afin "d'assurer le rayonnement international de la France et de sa cinématographie $\%$. Le Festival d'Avignon, lancé en 1947 par Jean Vilar, fait aussi figure de symbole, celui de la volonté d'après-guerre d'engager une politique de démocratisation de la culture.

Cette tendance s'est perpétuée à l'image du Printemps de Bourges, créé en 1977. ou des Francofolies de la Rochelle, lancées en 1985, même si les initiateurs se tournent dorénayant en priorité vers les collectivités locales.

\section{Les festivals de communication et de développement touristique}

Dans les années 1970 s'opère une prise de conscience des pouvoirs publics - inspirée par le succès d'expériences comme celles de Cannes ou d'Avignon - de la capacité des festivals à générer des retombées médiatiques et économiques. Elle se traduit par une nouvelle génération de festivals créés sur l'initiative des pouvoirs publics, dont l'objectif premier est de jouer un rôle de levier de développement économique ou de vecteur d'image. Cette prise de conscience n'est d'ailleurs pas à exclure du débat émergeant sur les enjeux économiques des activités culturelles qui trouvera sa réelle dimension dans les années 1980.
Bien que le projet culturel ne soit plus à l'origine de leur création, ces festivals n'en demeurent pas moins des événements qui exigent une qualité artistique, car seules l'originalité et la créativité du concept peuvent susciter l'attention des médias et

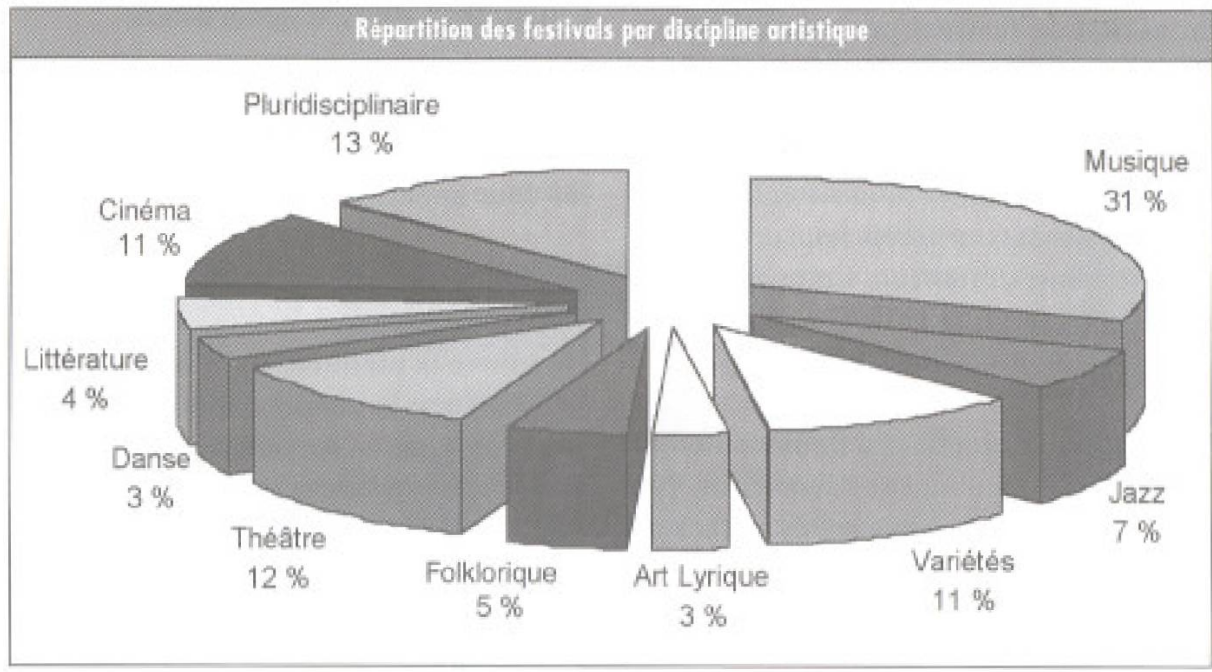

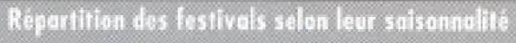

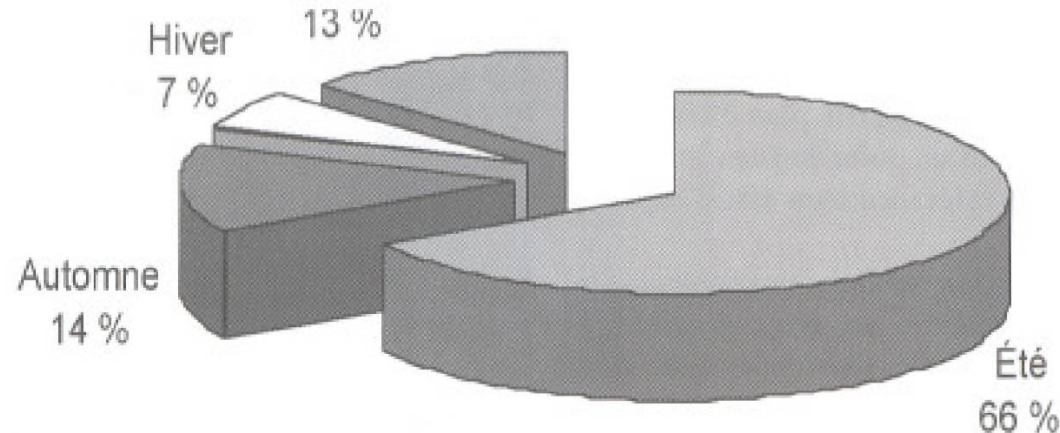

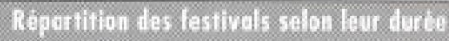

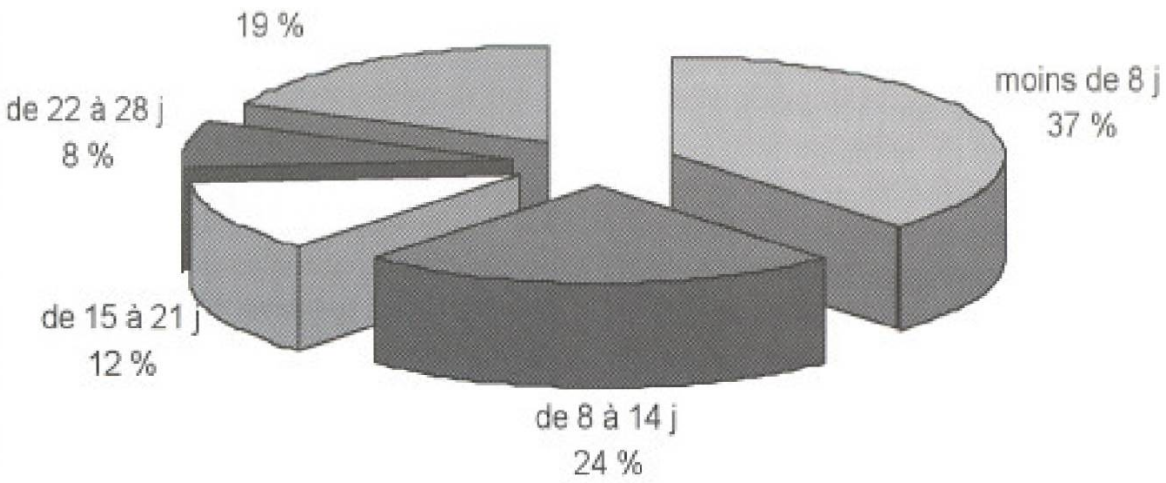




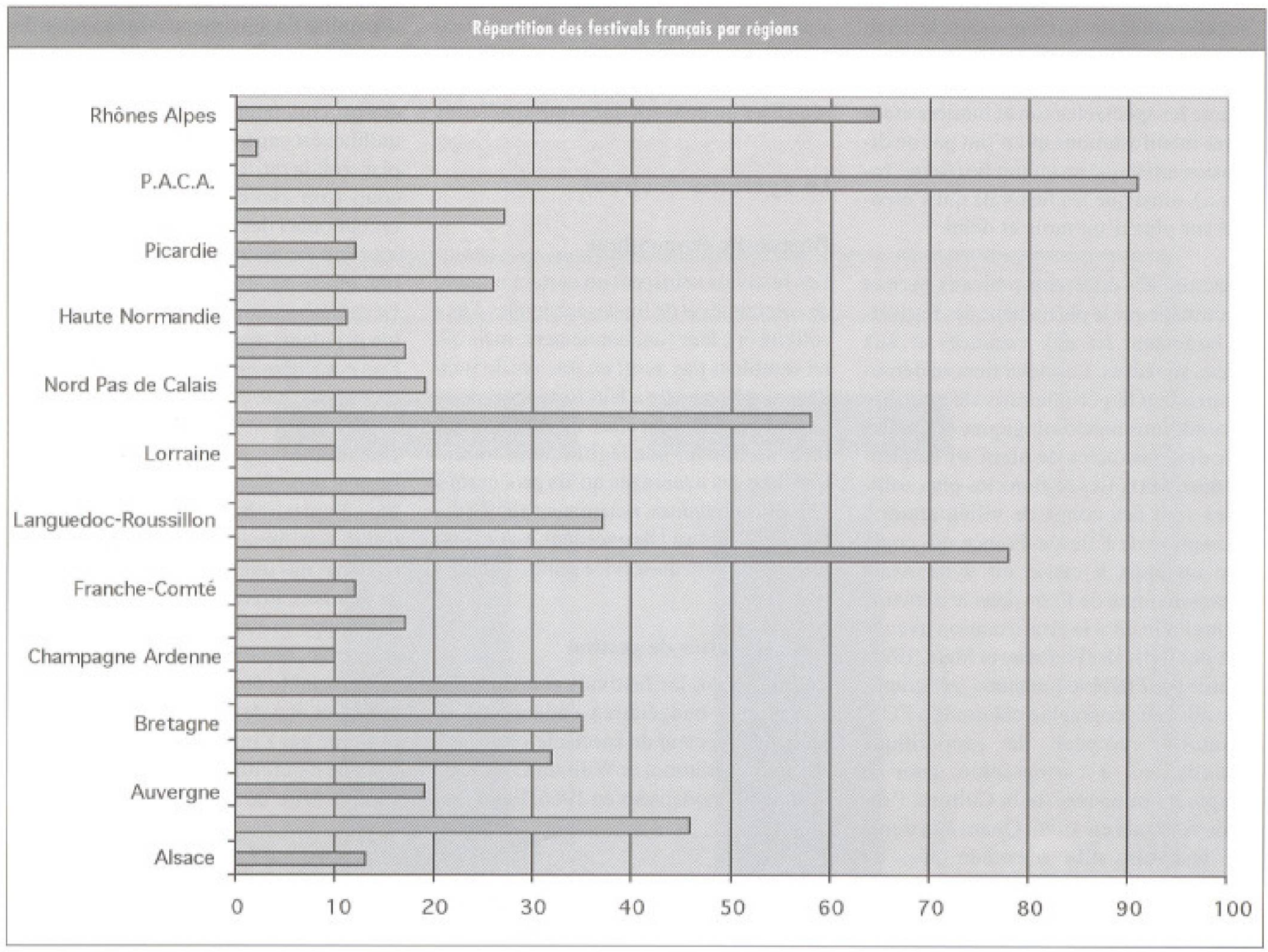

l'attraction des touristes et procurer, par conséquent, des retombées médiatiques et économiques.

Amorcée au début des années 1970 , cette tendance s'est réellement affirmée quand les lois de Décentralisation de 1982 ont donné aux collectivités locales la capacité financière de créer de tels événements. Les festivals sont alors apparus comme des atouts pour des collectivités locales désormais en concurrence quant à leurs attraits touristiques et culturels, et des moteurs pour l'économie locale dont elles avaient dorénavant la responsabilité́.

\section{Les festivals d'animation locale}

Face à la dégradation du contexte social et aux nouvelles pratiques de consomma- tion touristique, les pouvoirs publies locaux vont offrir des réponses qui se traduisent notamment en termes d'animation locale. L'organisation d'évenements apparaît done comme un moyen de satisfaire aussi bien les touristes que la population locale et, spécialement, ceux qui sont * exclus , financièrement ou géographiquement. Quant à la détermination du domaine, elle s'effectue en fonction des sensibilités des initiateurs, des associations ou des animateurs de rue, soit pour les arts, le sport ou le divertissement.

La priorité donnée au projet social ou à l'animation des touristes dans la création de ce type de festivals hypotheque parfois la qualitế artistique de l'événement. En effet, I'absence d'objectifs en termes de retombées médiatiques et économiques n'impose pas une qualité de la program- mation, contrairement aux festivals montés dans la tradition d'après-guerre.

Compte tenu de la faible sollicitation $\mathrm{fi}=$ nancière pour les collectivités locales, il semblerait que cette nouvelle génération de festivals à rayonnement local ait leur faveur, expliquant en cela la forte croissance de leur nombre depuis une quinzaine d'années.

\section{Le marché : une offre de plein aír}

Les différentes typologies présentées ici ont été obtenues d'après l'étude des guides culturels Festivals \& expositions 2000 et la Saison culturelle 2001 édités par le ministère de la Culture et recensant 692 festivals, de juin 2000 à mai 2001. Ces choix ont été dictés par un souci de fiabilité, car il s'agit des seules sources, à 
l'échelle nationale, qui fournissent le détail de la programmation des manifestations. Nous avons exclu certains événements, comme les spectacles son et lumière et les autres manifestations qui $n^{+}$ont pas de dimension artistique ou culturelle (foires, salons...), ainsi que les festivals qui s'étendent sur plus d'un mois et demi.

La lecture des différents tableaux permet de constater que le phénomène des festivals est largement lié aux vacances et aux congés scolaires. Les deux tiers se déroulent pendant la période estivale qui offre les conditions météorologiques optimales pour des spectacles de plein air en priorité musicaux. Les régions les plus sollicitées sont des zones de villégiature, à l'exception de l'Île-de-France qui constitue un biais à cause du dynamisme monopolistique de Paris dans le domaine culturel. On en a la confirmation avec la carte des festivals (Fourreau et Marc, 1997) réalisée par Frédéric Lamantia, géographe spécialisé en géographie culturelle à l' $\mathrm{Ob}$ servatoire européen de géopolitique d'Écully, qui a cartographié, pour le compte du ministère de la Culture, l'offre de festivals en 1996. Quant à la durée des festivals, elle n'excède pas, en moyenne, une dizaine de jours, mais l'on voit de plus en plus d'événements qui dépassent un mois. Un tel phénomène s'explique par le développement de festivals pluridisciplinaires qui illustrent la volonté des communes d'offrir de véritables mini-saisons culturelles en été.

Compte tenu des champs géographique et artistique désormais couverts par les festivals, il est devenu impératif pour eux de se démarquer des autres et de garantir l'originalité qui leur permettra d'attirer les médias et le public. Dans ce souci de différenciation, les festivals ont évolué, tant sur la forme que le fond, dans le sens d'une * dilution " de l'esprit du festival. La multiplication des festivals tend à a banaliser » l'événement: les festivals sont de plus en plus longs ou de plus en plus courts, de plus en plus éclatés géographiquement ; enfin, le terme « festival » est galvaudé et on lui préfere $*$ semaines de ou $\alpha$ rencontres de *. Quant au contenu, soit l'essence meme du festival, les observateurs s'accordent à dire que les audaces se raréfient, que la prise de risque disparaît et que les créateurs se contentent parfois de réutiliser un concept qui a réussi ailleurs.

\section{Le système festival}

\section{Diagnostic économique}

Les festivals subissent un certain nombre de contraintes et de limites inhérentes à leur activité et à leur fonctionnement, mais qui dence, si l'on se fie à leur forte croissance des années 1980. En effet, ils ont apparemment su trouver une légitimité économique dans les avantages qu'ils procurent à plusieurs domaines, notamment auprès des pouvoirs publics; ils excèdent, en ce sens, le simple cadre du service public culturel.

\section{Les difficultés de gestion}

Tout d'abord, les festivals souffrent des contraintes budgétaires endémiques et propres au secteur du spectacle vivant que William J. Baumol et William G. Bowen (1966) ont modélisées en 1966. Ils ont fait ne semblent pas avoir eu une réelle inci-

apparaître l"accroissement inéluctable des coûts de production des structures de diffusion de spectacles vivants. Une hausse des prix qui pourrait compenser ce déséquilibre est cependant impossible, car elle placerait la tarification à un niveau beaucoup trop élevé. De plus, le caractère exceptionnel des festivals pousse toujours les programmateurs à proposer une qualité, qui se paye généralement très chère. Le caractère temporaire des festivals semble d'ailleurs accroittre ce phénomène, si l'on considere que le niveau d'autofinancement se situe en moyenne de 20 a $40 \%$.

Une telle situation rend les festivals largement dépendants des apports financiers. Mais contrairement à beaucoup d'autres pays, notamment nord-américains, le mécénat qui qualifie la participation des entreprises privées n'est pas beaucoup développé en France. Dans la conscience collective, le parrainage d'activités culturelles semble être un mariage contre nature. Cet état de fait se trouve d'ailleurs renforcé par l'interdiction pour des marques de cigarettes et de boissons alcoolisées de faire de la publicité.

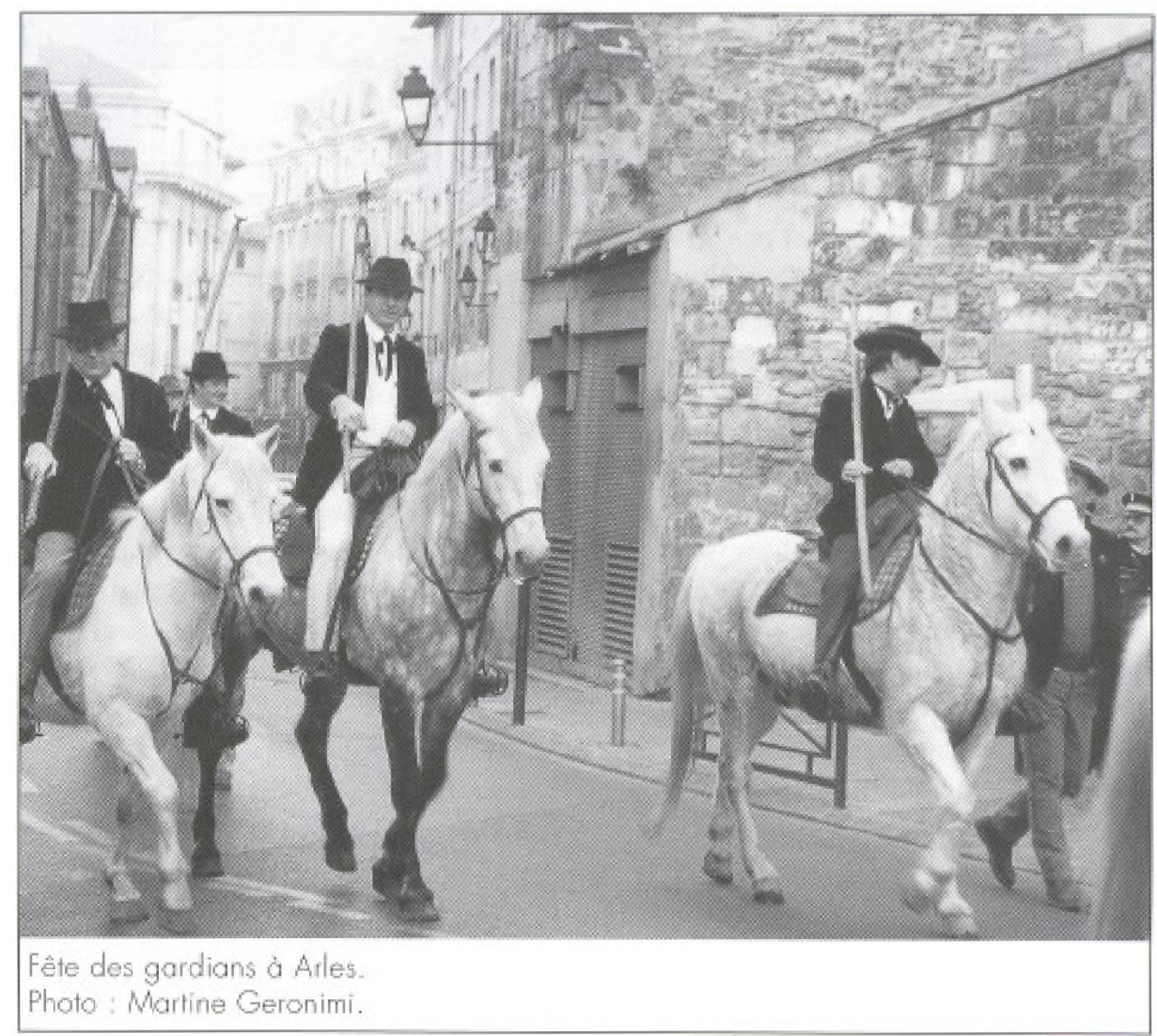


Par ailleurs, en France, les partenaires financiers qui sont principalement les pouvoirs publics rechignent à s'engager sur plusieurs années, contraignant les responsables à sortir chaque année leur bâton de pèlerin pour aller convaincre des mécènes. Ils doivent aussi veiller à ce qu'on ne leur impose pas une contrepartie trop pesante qui mettrait en péril la liberté des choix artistiques du programmateur.

Finalement, une autre conséquence de cette précarité du montage financier est le recours à un personnel souvent bénévole et peu qualifié alors que ce type d'activité nécessite des compétences professionnelles. D'autant que beaucoup de responsables du monde des arts et de la culture ont une certaine répugnance pour les questions relatives a la gestion.

Au-delà de ces problèmes de fonctionnement interne, les festivals subissent des contraintes de nature différente davantage liées au contexte : il s'agit de la concurrence sans cesse croissante depuis les vingt dernières années qui a provoqué une sáturation de l'offre pour les publics, les médias et les partenaires financiers qui ne peuvent plus répondre à toutes les sollicitations.

Le manque de fiabilité dû à des programmations toujours tardives des festivals n"incite pas les opérateurs touristiques à créer et à promouvoir des produits autour d'eux, d'autant que les taux de marge escomptés sont rélativement faibles.

Quant au contexte politique, il est évidemment ici très important. Pendant les années 1990 , les pouvoirs publics ont donné la priorité à la lutte contre le chômage et à l'exclusion des minorités sociales au détriment des budgets alloués à la culture. Les festivals ont subi de plein fouet la baisse des subventions publiques à l'origine d'une période de rationalisation relative à cette activité.

\section{Les missions dévolues}

Dans le domaine purement artistique, le festival est un lieu propice, plus que les institutions permanentes, à la cré̉ation artistique et à la prise de risque, ces dernières étant inhểrentes à une programmation de qualité. Ils sont aussi des éléments moteurs dans la découverte de nouveaux talents et sont les lieux de prédilection pour la présentation d'œuvres en avant-première, comme au Festival d'Avignon qui offre la primeur de certains spectacles avant le démarrage de la saison ou dans les festivals de cinéma qui proposent les films en général avant leur sortie. En ce sens, les festivals permettent, entre autres, la rencontre entre des producteurs d'œuvres qui ont la possibilité de montrer leur création et des programmateurs qui viennent faire leur sélection pour la saison à venir. Toujours en matière de promotion, les festivals favorisent l'émergence de genres artistiques écartés des réseaux de diffusion traditionnels, en permettant d' obtenir une reconnaissance publique par la médiatisation de l'événement et de satisfaire leur public.

Sur le plan des problématiques d'ordre culturel, les festivals sont apparus comme des vecteurs de développement efficaces. En matière d'aménagement du territoire, ils contribuent à la décentralisation culturelle en apparaissant, pour des petites localités isolées n'ayant pas de structures permanentes, comme le seul moyen d'assister à des spectacles donnés dans les grandes villes, Quant à ces localités certaines d'entres elles -, le festival est à l'origine d'une véritable dynamique pour la politique culturelle municipale (notamment grâce au développement d'équipements culturels). Mais le festival reste avant toụt un élément majeur de promotion d'un genre artistique en lui offrant parfois un territoire. Ainsi, Avignon est devenue la patrie du thêtre et Cannes celle du cinéma.

Phénomène bien connu en Amérique du Nord, les études d'impacts économiques sont apparues en France il y ane quinzaine d'années dans le but de justifier une certaine rentabilité économique, surtout auprês des collectivités locales. Bien que leur fiabilité ait été remise en question depuis, il apparaît clairement que les festivals constituent un moteur pour le tissu économique local. Les retombées que procure les activitếs liées à un festival profitent en premier aux entreprises du secteur touristique (transport, hébergement, restauration et animation). Les dépenses des festivaliers ainsi que les budgets \& voyage * réservés aux artistes et à leurs consommations éventuelles constituent un accroissement non négligeable de leur activité. Mais si l'augmentation du chiffre d'affaires des acteurs touristiques est bien réelle, les répercussions sur l'emploi restent aléatoires, car il s'agit plutôt d'emplois saisonniers que d'emplois permanents requérant des qualifications élevées.

Outre l'aspect économique, le secteur touristique bénéficie des retombées produites par l'activité des festivals, particulièrement sur le plan de la valorisation. La ville organisatrice dispose d'une capacité d'attraction de touristes grâce à un festival qui la glorifie d'une image de destination touristique. Les prolongements ne sont d'ailleurs pas uniquement visibles pour les publics touristiques puisque des congrès sont souvent organisés en même temps.

De plus, sa fonction d'animation confere à la ville une atmosphère de fête qui accroît son attrait touristique. On constate aussi, comme dans l'étude sur les festivals d'été de Montréal (Arehambault et âl., 1996), que les festivals incitent les touristes à prolonger la durée de leur séjour. Étant des publics sensibles aux pratiques culturelles, ils ont plutôt tendance à en profiter pour visiter le patrimoine local (musées, monuments historiques...).

De leur capacité intrinsèque à attirer l'attention des médias, liée à leur caractère événementiel, les festivals tirent une offre de services tout al fait pertinente en termes de communication. Outre la possibilité d'obtenir des invitations gratuites, e'est en tant que support de communication qu'ils apparaissent les plus pertinents pour certains commanditaires.

La couverture médiatique d'un festival accroît la notoriété d'une localité, en lui offrant une publicité que son budget de communication ne lui permettrait sans doute pas d'atteindre. Les festivals sont 
en effet des vecteurs d'image, qui ont poussé les collectivités territoriales (départements et régions) à développer leur communication autour de ce type d'événement. L'image que véhicule un festival est celle d'un dynamisme culturel de la collectivité locale dont les effets se traduisent différemment suivant les cibles. Auprès des touristes, elle doit promouvoir la ville comme destination touristique en communiquant une atmosphère de fête et de convivialité, alors que pour l'investisseur. en quête d'un site d'implantation de son entreprise, l'image d'un bouillonnement culturel est souvent un élément déterminant. Enfin, pour l'administré, elle doit témoigner de l'engagement de la collectivité en faveur de la culture, sensible notamment auprès des jeunes.

Par ailleurs, en ce qui concerne les annonceurs privés, les festivals, au même titre qu'une exhibition sportive, sont des supports de communication privilégiés pour promouvoir une marque ou un produit. Leur intervention se limite en général à la présence de logos sur les billets et les autres supports publicitaires et, plus rarement, dans la dénomination du festival. Outre la dimension purement commerciale, les festivals offrent aussi aux entreprises un service d'image qui répond aux objectifs de leur politique de communication, comme dans le cas des entreprises du secteur bancaire et des assurances qui participent financièrement à des activités non lucratives pour redorer une image quelque peu déficitaire,

On observe enfin que l'activité des festivals a une réelle action sur le plan de la dynamique sociale. Sa principale spécificité en la matière tient dans leur capacité à mobiliser la population locale, dont témoigne un fort taux de bénévolat. La participation bénévole se trouve d'ailleur's aussi bien sur le plan de l'organisation technique des festivals que de la production artistique. Ce phénomène s'explique en grande partie par le caractère plutôt valorisant des activités artistiques et culturelles. La participation, généralement dans une ambiance de fête, à un événement d'utilité collective et médiatisé, de surcroit, procure une certaine satisfaction et donne aux résidants une certaine conscience d'eux-mêmes en tant que citoyens. Le phénomène se trouve largement amplifié quand il s'agit pour la population de présenter avec une grande fierté son propre patrimoine culturel (musiques et danses traditionnelles, spécialités culinaires, us et coutumes locales...) par le biais d'événements folkloriques.

La forte mobilisation de la population locale autour de ces festivals permet de resserrer les liens sociaux de communautés ou de populations de plus en plus isolées, souvent en proie à l'exclusion. Pour des ruraux, un festival est l'occasion de garantir une cohésion sociale que la désertification tend à dissoudre. L'exemple du Festival de la Bogue d'Or en Bretagne, basé sur la culture communautaire, montre que les activités de fêtes fournissent de nouvelles formes de convivialité assurées traditionnellement par l'activité agricole, qui a pratiquement disparu.

\section{Perspectives ef orientations}

\section{La mobilisation des acteurs}

Pour pallier les difficultés financières endémiques des festivals, l'engagement financier des acteurs bénéficiant de ces retombées est indispensable, particulièrement sous la forme d'une contractualisation pluriannuelle. La participation aux festivals ne se limite cependant pas uniquement à la dimension financière; elle se traduit aussi en termes de contribution technique, voire de cautionnement moral. C'est le cas notamment de la participation financière de l'État car si, quantitativement, le soutien financier étatique, orchestré principalement par le ministère de la Culture, peut apparaître comme mineur, il n'en demeure pas moins indispensable au milieu artistique. La contribution du ministère de la Culture agit en effet comme un label de qualité compte tenu des critères retenus pour l'attribution des subventions, ces dernières pouvant faciliter l'obtention d'autres participations des collectivités locales.

En effet, les directions régionales des affaires culturelles, organes déconcentrés du ministère de la Culture en région de- puis 1992, sont les seules à pouvoir accorder les subventions de l'État aux festivals. Dans une moindre mesure, les sociétés civiles professionnelles aussi encouragent la création en soutenant des manifestations qui prennent des risques artistiques et qui représentent des vitrines de la France.

Mais ne nous y trompons pas, $c^{\prime}$ est bien auprès des pouvoirs publics locaux qu'il faut trouver la première forme de soutien d'un festival. Rappelons ici que la prise de conscience - notamment des communes - de l'utilité des festivals dans la promotion de leur collectivité est le facteur principal qui explique l'incroyable développement de ces événements depuis une vingtaine d'années. Désormais, aucun projet, de quelque nature que ce soit, ne peut se réaliser sans l'accord, au minimum tacite, de la commune d'accueil.

Cependant, si la ville est devenue le premier partenaire financier des festivals, leur réussite dépend d'une bonne intégration dans l'environnement politique, culturel, touristique et social. Tout d'abord, on constate souvent une absence de lien entre les festivals et les institutions musicales ou thêtrales de la ville d'accueil, d'où un sentiment de «parachutage * plus ou moins bien vếcu. On relève également une absence de liaison entre les disciplines artistiques et les festivals qui se déroulent dans une même ville. Il faut par conséquent veiller, pour les villes en particulier, à mieux insérer les festivals dans leur environnement culturel, de manière à ce qu'ils valorisent et renforcent les liens avec les équipements culturels permanents.

L'intégration des objectifs du festival dans la politique touristique des collectivités locales passe par une réflexion sur la durée et la date de la manifestation, pouvant être déterminées en fonction des particularités de la saison touristique du site festivalier. Quand les collectivités locales en sont les instigatrices, il arrive aussi que les thèmes des festivals soient déterminés en fonction de la valorisation du patrimoine architectural local.

S'agissant de la communication institutionnelle, même si les actions des collectivi- 


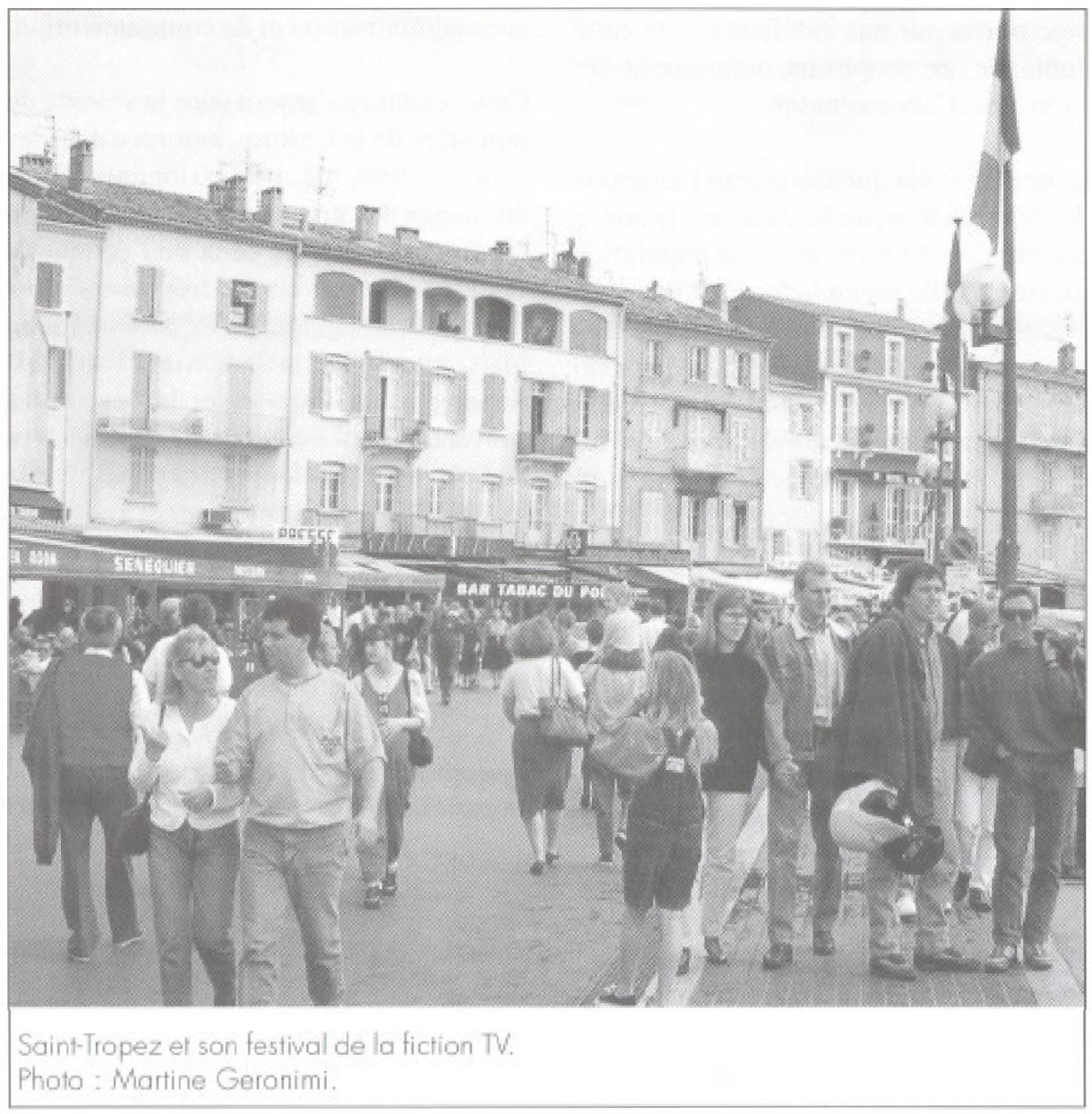

tếs locales étaient menées jusqu'à présent au coup par coup, beaucoup d'entre elles possèdent désormais une réelle politique de communication. Comme on l'a vu, les festivals sont d'ailleurs apparus, pour un bon nombre d'entre elles, comme un outil particulièrement efficace notamment, pour sortir de l'anonymat.

Le recours au soutien des entreprises privées n'est pas à ignorer. Apparu dans les années 1970 comme un financement complémentaire de la politique culturelle, le mécénat s'est développé dans les années 1980, particulièrement grâce aux mesures d'incitations fiscales de juillet 19871.

Le mécénat reste cependant encore peu pratiqué en France, comparé à d'autres pays. Ce point se vérifie dans le financement des festivals, où la part du mécénat s'avère relativement faible, puisqu'elle représente en moyenne moins de $14 \%$ du budget d'un festival, alors que les subventions publiques s'élèvent à environ $50 \%$.
Pur ailleurs, le mécénat de festivals apparait sélectif, excluant ceux dont le rayonnement est trop grand pour intéresser des PME, mais insuffisant pour de grandes entreprises. En ce sens, la constitution de groupes d'entreprises-mécènes composés d'acteurs économiques locaux offre des possibilités de financement des festivals.

Même si la commercialisation touristique des festivals apparaît problématique pour les opérateurs du tourisme qui ont besoin d'une programmation sûre planifiée plusieurs mois à l'avance, la collaboration entre les deux n'est pas à négliger. Les voyagistes intègrent volontiers les festivals, non pas par rapport à leur programmation, mais comme élément complémentaire d'animation dans un produit global. Quant aux produits touristiques crés autour de l'événement, ils sont aussi rares que les tours opérateurs spécialisés. Pour pallier cette carence, les responsables de festivals prennent de plus en plus I'initiative et conçoivent eux-mêmes des produits de ce type pour les proposer ensuite à un voyagiste.

Le tissu socio-économique local n'est enfin pas non plus à négliger car l'on sait que la participation de bénévoles, bien que sujette à caution parfois, constitue souvent l'une des clés de la réussite d'un festival.

\section{La professionnalisation du secteur}

Outre la mobilisation des acteurs, surtout au plan local, dorénavant indispensable pour la continuité des festivals, il reste aux responsables à susciter l'engagement de leurs partenaires potentiels et à faire preuve de plus de rigueur dans la gestion de leur manifestation. Un soin tout particulier doit être accordé à la détermination des caractéristiques techniques des festivals car elles participent à l'originalité du concept de l'événement. De fait, la négligence de certains paramètres (date, durée, dénomination, choix des sites, signalisation, accueil ou tarification) a parfois conduit à l'échec d'un événement, alors que la qualité artistique était au rendez-vous.

Sous l'effet de contraintes financières lourdes, les festivals ont cherché à accroitre leurs sources de revenus annexes, en développant de nouvelles activités, aussi bien sous la forme de services que de produits. Il peut s'agir de la vente de programmes, de posters, d'affiches, de souvenirs et de tout autre produit dérivé, l'édition de disques ou de livres, par exemple, ou la cession de droits de diffusion médiatique.

La location de stands publicitaires ou d'espaces réservés dans le cadre de journées professionnelles ou de salons organisés par les responsables de festivals est une source de revenus non négligeable.

La réussite d'un festival dépend en grande partie de la capacité du directeur artistique à offrir un programme original, savante combinaison entre les répertoires, les interprètes et les lieux. La qualité artistique de la programmation apparaît en ce sens comme le premier facteur de fidélisation du public. 
Offrir toujours davantage, innover ou découvrir de nouveaux talents, relèvent d'un travail de spécialistes, dont la liberté de programmation est une condition sine qua non. Cette marge de liberté se voit désormais considérablement réduite à cause de la multiplication des acteurs dont les aspirations vont davantage vers le vedettariat.

La programmation de jeunes createurs attire généralement moins de public et génère par conséquent moins de retombées économiques que la présence d'artistes confirmés. À l'inverse, le manque d'originalitế crếatrice d'une programmation trop traditionnelle réduit la qualité, limitant la notoriété d'un festival et, par voie de conséquence, les retombées médiatiques pour la ville.

Ainsi, la direction artistique se présente comme un arbitrage entre des logiques artistique et marchande de plus en plus interdépendantes. C'est pourquoi la plupart des directeurs de festivals, conscients de cette situation, ne conçoivent plus la présentation de spectacles de création sans la présence de valeurs sûres.

La recrudescence des activités culturelles et ludiques, ces dernières années, a poussé les responsables des festivals à accroître la promotion de leur événement en ayant recours à des études de publics. En effet, la connaissance du profil socioéconomique, de l'origine géographique et des motivations du public sont indispensables à la détermination de campagnes de promotion efficaces et de supports de communication pertinents. Sur ce point, on remarque que, pour des festivals à faible notoriété, souvent jeunes, le public venu spécialement pour l'événement représente une part assez faible, car il est sensible à d'autres variables, liées notamment aux motivations touristiques. La promotion devrait en ce sens adopter une démarche plutôt informationnelle à rayonnement local, afin de stimuler une demande déjà présente sur le site. Dans le cas d'événements de renom, par ailleurs, dont la qualité et la pérennité assurent l' intérêt du public, les actions de promotion devraient davan- tage porter sur une fidélisation de cette demande, en proposant notamment des formules d'abonnement.

Le dernier point qui doit retenir l'attention des responsables de festivals est la bonne acceptation du festival par la population locale, car elle prend la forme d'une légitimation, pour deux raisons. D'une part, la population locale estime logiquement avoir un droit de regard sur l'évếnement, dans le sens où il est subventionné par ses impôts, et, d'autre part, elle doit supporter les nuisances produites par l'animation et l'afflux de touristes.

On ne peut exclure la population locale de la fête sans risquer de faire des mécontents parmi les contribuables locaux. À Cannes, où le festival est réservé aux seuls professionnels, beaucoup de résidants voient, en effet, d'un très mauvais cil qu'on leur refuse I'accès au Palais des festivals, un équipement construit par la ville. De plus, négliger l'intégration du festival auprès de la population locale peut dans certains cas conduire à l'échec, surtout s'il relève d'un * parachutage culturel $»$. En effet, le public local peut bouder l'événement, excluant une grande partie du public potentiel du festival, ou encore l'accueil inhospitalier de la population peut dissuader les touristes festivaliers.

Outre tenir la population locale informée et valoriser auprès d'elle les nombreuses retombées locales, il convient d"ouvrir le festival par l'intermédiaire d'actions de promotion et de sensibilisation, surtout si la thématique est difficile d'accès. La création de classes ou de cours spécialisés dans les établissements scolaires et l"intervention d'artistes témoignent d'ailleurs de cette tendance.

Parmi les compétences dévolues aux responsables des festivals, qui sont désormais indispensables pour assurer la viabilité de leur manifestation, beaucoup relèvent des techniques de gestion et d'évaluation. Car, outre la mise en place d'actions, un suivi est nécessaire pour garantir, d'une part, la satisfaction du public en termes d'accueil, de tarification et de programmation et, d'autre part, l'efficacité des politiques de commercialisation et de communication.

Cette tendance s'inscrit dans la volonté du ministère de la Culture, amorcée dans les années 1980, de professionnaliser les domaines des arts et de la culture par un rapprochement avec ceux de l'économie et de la gestion, considérés jusqu'alors comme incompatibles, voire antinomiques. Mais, auldelà de l'incitation de l'État, c'est surtout la concurrence et la baisse des subventions qui a conduit les responsables de festivals à développer des compétences dans la recherche de mécènes et à accroître l'efficacité dans la gestion de leur activité.

Luc BENITO, consultant en ingénierie culturelle et touristique, responsable pédagogique à l'Université d'Aix Marseille I, est auteur de a Les festivals en France: marchés, enjeux et alchimie * publié aux Editions I'Harmattan en 2000.

\section{Note}

1 Les dispositions de la loi du 23 juillet 1987 prévoient des déductions pour les entreprises, de l'ordre de 2-3 \%o de leur chiffre d'affaires, selon l'organisation bénéficiaire, pour les dépenses de mécénat ou les dons sans contrepartie.

\section{Bibliographie}

Archambault, Michel et al. (1996), Etude du positionnement touristique des quatre grands festívals d'élé de Montréal, Montréal, Université du Québec à Montréal (UQAM).

Baumol, William J., et William G. Bowen (1966), Performing Arts. The Economic Dilemma, Cambridge, Massachusetts, Twentieth Century Fund.

Benito, Lac (2001), Les festivals en France: marchés, enjeut el alchimie, Paris, L'Harmattan.

Fourreau, Éric, et Marc, Nicolas (1997), « Les festivals $\%$, La Scène, $\mathrm{n}^{\circ}$ 7, décembre, Caen, p. $17-32$. 